\title{
O USO DO FORNO DE MICROONDAS NA SÍNTESE ORGÂNICA EM FASE SÓLIDA
}

\author{
Cedric Stephan Graebin e Vera Lucia Eifler-Lima*
}

Faculdade de Farmácia, Universidade Federal do Rio Grande do Sul, Av. Ipiranga, 2752, 90610-000 Porto Alegre - RS

Recebido em 21/10/03; aceito em 13/4/04; publicado na web em 9/8/04

\begin{abstract}
THE USE OF MICROWAVE OVENS IN SOLID-PHASE ORGANIC SYNTHESIS. Solid-phase organic synthesis (SPOS) has been considered the main strategy for the construction of combinatorial libraries, because its simplicity leads to faster synthetic procedures. In addition to that, a series of reports in the specialized literature show great advantages in the use of microwave activation, when compared to classical heating, for instance: shorter reaction times, in some cases from several hours to a few minutes, increase of selectivity and product yields, energy economy and reduction and/or elimination of solvent. This review describes the use of microwave ovens/reactors in solid phase organic synthesis, describing the advantages, equipment and reactions using both techniques.
\end{abstract}

Keywords: microwave reactor; solid-phase organic synthesis; combinatorial chemistry.

\section{INTRODUÇÃO}

A Síntese Orgânica em Fase Sólida (SOFS), criada por Bruce Merrifield no início dos anos $60^{1}$, popularizou-se na última década devido a suas vantagens, a saber: a grande facilidade da purificação dos produtos intermediários, que consiste de filtrações com vários solventes - e sua capacidade de automatização, que permite ao químico a síntese de um grande número de derivados em um tempo relativamente curto. Contudo, esta técnica apresenta ainda algumas desvantagens, tais como o trabalho com meios heterogêneos e os longos tempos de reação empregados, causados muitas vezes pelas dificuldades de monitoramento in situ das reações. Estes são ainda alguns dos desafios que os químicos orgânicos devem vencer para dominar a SOFS.

Mesmo que existam ainda desafios a serem vencidos, a popularização da Química Combinatória ${ }^{2}$ como ferramenta válida e útil para o descobrimento de novas moléculas bioativas só ocorre porque a SOFS é a principal estratégia de síntese de quimiotecas combinatoriais.

Muitos químicos têm utilizado com sucesso o reator de microondas como fonte de ativação de reações químicas em SOFS, objetivando acelerar ainda mais a obtenção dos produtos. Desta forma, conseguiu-se também, como será demonstrado no decorrer deste trabalho, tempos menores e rendimentos maiores nas reações.

Desde 1986 existem relatos na literatura que apontam o uso do reator de microondas como fonte de aquecimento de reações químicas, sempre ressaltando a obtenção de tempos de reação menores e rendimentos superiores àqueles obtidos quando a fonte de aquecimento era convencional ${ }^{3}$, isto é, aquecimento com o uso de manta de aquecimento ou chapa-quente.

O objetivo deste trabalho é, principalmente, discutir as reações químicas em SOFS nas quais essa estratégia foi aplicada com sucesso.

\section{REVISÃO DA LITERATURA: REAÇÕES EM FASE SÓLIDA ASSISTIDAS POR MICROONDAS}

Nos últimos 10 anos vários trabalhos foram publicados na literatura especializada, unindo as técnicas da Síntese Orgânica em Fase

*e-mail: eifler@farmacia.ufrgs.br
Sólida e o uso do reator de microondas, embora a grande parte dos trabalhos publicados na área tenha sido feita com a técnica clássica de síntese orgânica, isto é, a síntese em solução, muito bem documentada na literatura ${ }^{4}$. A seguir, as publicações nas quais o uso da SOFS foi combinado com o emprego de reatores de microondas.

\section{Reações de acoplamento}

A reação de acoplamento de uma molécula de interesse a um suporte sólido (ou resina) é importantíssima, uma vez que é a partir desta reação que se inicia qualquer trabalho com a técnica de SOFS. Os tempos de reação relatados na literatura, utilizando-se de aquecimento convencional, variam de 13 a $18 \mathrm{~h}^{5}$. Empregando um reator monomodo de MO, Kappe e Stadler ${ }^{5}$ acoplaram 33 ácidos (aromáticos, derivados do ácido benzóico) à resina Merrifield em presença de base $\left(\mathrm{Cs}_{2} \mathrm{CO}_{3}\right)$, em um tempo médio de $5 \mathrm{~min}$ (Esquema 1).

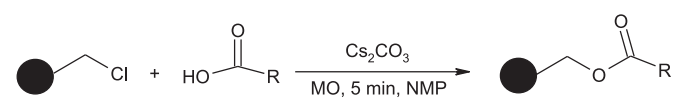

NMP: N-metilpirrolidona

Esquema 1. Acoplamento de ácidos à resina Merrifield

Kappe et al., em outro trabalho ${ }^{6}$, acoplaram na resina Wang o ácido 4-cloro-3-ceto-butílico em 15 min, utilizado como produto de partida na síntese de diidropirimidonas bicíclicas (Esquema 2).

Berthena-Raboin et al., numa rota sintética visando a síntese de derivados 5-carboxamido- $N$-acetiltriptaminados ${ }^{7}$, compararam os tempos de acoplamento do produto de partida, o ácido-3-iodo-4aminobenzóico à resina Rink Amide em um reator de microondas (monomodo, com potência de $300 \mathrm{~W}$ ) e em temperatura ambiente, observando que em MO a reação ocorreu em 3 min, com $85 \%$ de rendimento, contra 24-48 h à temperatura ambiente, com $62 \%$ de rendimento (Esquema 3)

Kodadek et al., em uma rota de síntese de peptóides ${ }^{8}$, acoplaram o ácido bromoacético na resina Tentagel Rink em 30 s (Esquema 4), tempo total feito com duas exposições do meio reacional às microondas de $15 \mathrm{~s}$ cada, de modo a não se elevar muito a temperatura da 


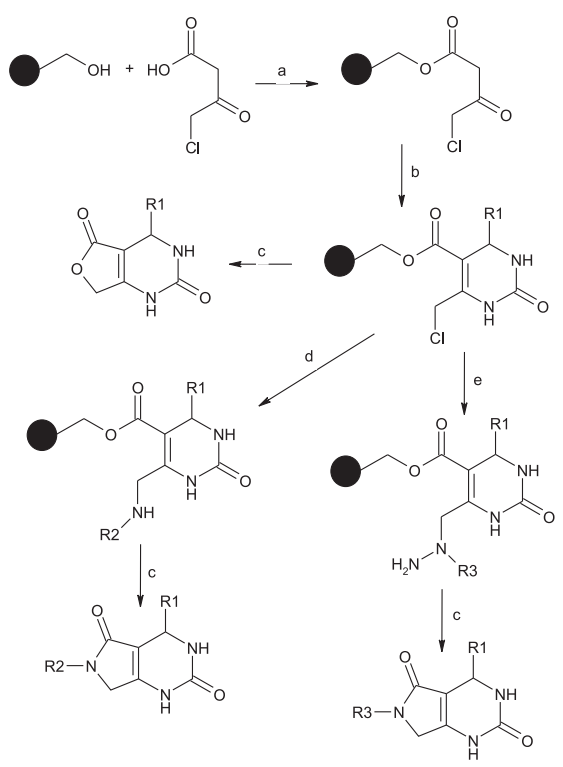

Condições: a) 1,2-diclorobenzeno, $\mathrm{MO}\left(170^{\circ} \mathrm{C}\right), 15 \mathrm{~min}$; b) $\mathrm{R}_{1} \mathrm{CHO}$, uréia, dioxano, $\mathrm{HCl}, 70^{\circ} \mathrm{C}$, "overnight", depois $\mathrm{HCl}$ conc., temp. ambiente, $\left.5 \mathrm{~min} ; \mathrm{c}\right) \mathrm{N}, \mathrm{N}$ dimetilformamida (DMF), $\mathrm{MO}$, (150 ou $\left.\left.200{ }^{\circ} \mathrm{C}\right) 10 \mathrm{~min} ; d\right) \mathrm{R}_{2} \mathrm{NH}_{2}, \mathrm{DMF}, 50 \mathrm{ou} 70{ }^{\circ} \mathrm{C}$, "overnight" e e) $\mathrm{R}_{3} \mathrm{NHNH}_{2}, \mathrm{DMF}$, temp. ambiente, $30 \mathrm{~min}$.

Esquema 2. Síntese de diidropirimidonas bicíclicas
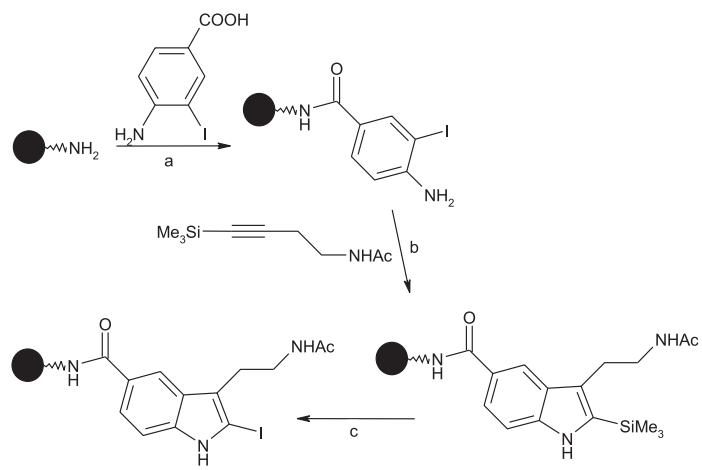

Condições: a) N,N,N',N'-tetrametil-O-(benzotriazol-1-il)-urônio tetrafluoroborato (TBTU), hidroxibenzotriazol (HOBT), trietilamina, 4-diaminopiridina (DMAP), dioxano, $M O$, 3 min ou refluxo, $24 h$; b) $P d(O A c), P P h_{3}, L i C l, N a O A c, ~ N-N-$ dimetilanilina (DMA), MO, 13 min ou $24 \mathrm{~h}, 100^{\circ} \mathrm{C}$; c) N-iodo-succinimida (NIS), diclorometano (DCM), MO, 14 min ou refluxo $24 \mathrm{~h}$

Esquema 3. Síntese de derivados 5-carboxamido- $N$-acetiltriptaminados

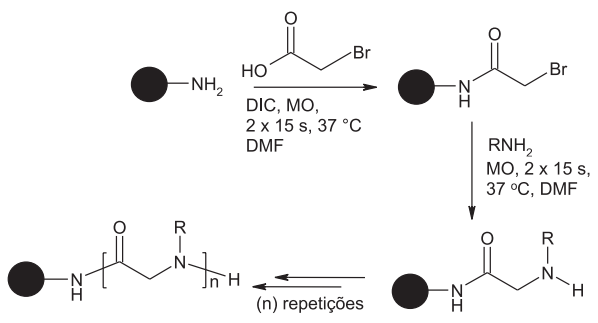

DIC: diisopropilcarbodiimida reação. Este controle "manual" foi necessário pelo fato de que o microondas utilizado era um forno doméstico, com potência nominal de $1000 \mathrm{~W}$. A pureza obtida sob MO foi de $60-90 \%$, contra $63-80 \%$ à 37 ${ }^{\circ} \mathrm{C}$ e $25-70 \%$ à temperatura ambiente. Observa-se que, embora as resinas constituídas de polietilenoglicol, como a resina Tentagel, sejam mais "frágeis" que as constituídas de poliestireno/divinilbenzeno, elas permanecem íntegras mesmo quando expostas ao aquecimento muito rápido proveniente das microondas.

Stadler et al. ${ }^{9}$ acoplaram a metilamina na resina Merrifield em 5 min. Este trabalho, realizado em escala piloto com um reator multimodo, foi efetivado utilizando-se a água como solvente (segundo os autores, a solução de metilamina disponível era $40 \%$ em água), um solvente não recomendado para a SOFS, principalmente com resinas de poliestireno (Esquema 5).

$$
\curvearrowright \underset{\mathrm{Cl}}{\mathrm{MO}, 150^{\circ} \mathrm{C}, 5 \mathrm{~min}} \curvearrowright \mathrm{NHMe}_{\mathrm{MHH}}
$$

Esquema 5. Acoplamento da metilamina na resina Merrifield

Recentemente a reação de acoplamento do acetoacetato de etila à resina Merrifield, em meio alcalino e sem solvente, foi realizada com sucesso, não ocorrendo a degradação do material de partida ${ }^{10}$, utilizando-se um forno doméstico como reator. Esse método mostrou-se excelente para o acoplamento desse éster à resina Merrifield (Esquema 6).

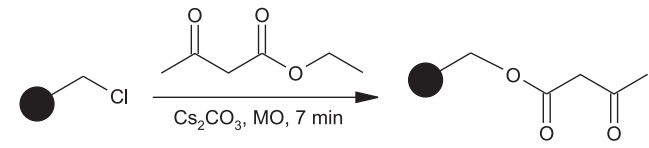

Esquema 6. Acoplamento do acetoacetato de etila à resina Merrifield

\section{Reações de clivagem}

A reação de clivagem, isto é, a liberação do produto de interesse que está preso à resina é, juntamente com o acoplamento, etapa fundamental nas reações de SOFS. Isto porque o método de clivagem escolhido deve fornecer o produto planejado com o máximo de pureza e rendimento. A escolha de um método inadequado na clivagem pode levar à degradação ou formação de subprodutos. A clivagem pode ser realizada de diferentes maneiras, dependendo da resina com a qual se está trabalhando e também da estrutura do produto desejado. Utilizando-se as resinas Merrifield e Wang, entre as mais utilizadas atualmente, em que geralmente há ligação éster entre a resina e o produto, a clivagem pode ser feita em meio ácido (com TFA ou HF) ou básico, na maioria das vezes com metóxido de sódio.

Hlasta e Deng ${ }^{11}$ realizaram a clivagem com uma resina cujo grupo ligante era um carbamato. A clivagem, feita com auxílio de um nucleófilo $(\mathrm{Nu})$ foi realizada em 5 min em um reator monomodo de MO com a obtenção do produto final com pureza de $65-95 \%$ e 80 $87 \%$ de rendimento, contra $1 \mathrm{~h}$ de aquecimento convencional, com $55-95 \%$ de pureza e $78-86 \%$ de rendimento (Esquema 7 ).

Kappe et al. ${ }^{6}$, na rota de síntese de diidropirimidonas bicíclicas, realizaram três clivagens em produtos análogos sob as mesmas con-

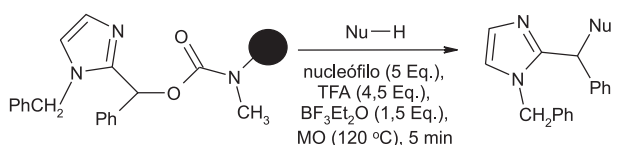

TFA: ácido trifluoracético 
dições, em 10 min sob MO (Esquema 2, reação “c"), obtendo purezas superiores a $95 \%$ e rendimentos variando de $14-77 \%$.

\section{Reações de condensação}

A Condensação de Knoevenagel foi realizada em microondas ${ }^{12}$, utilizando-se o éster nitroacético acoplado à resina Wang em oposição a diversos aldeídos. Observou-se a formação dos produtos num tempo médio de 20 min (Esquema 8).

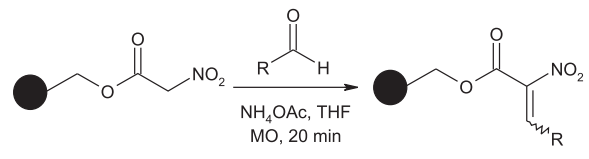

Esquema 8. Condensação de Knoevenagel

\section{Reações de substituição}

Song et al. ${ }^{13}$ realizaram várias reações de substituição nucleofílica na resina Merrifield, em um reator monomodo, com o objetivo de funcionalizá-la, obtendo bons resultados (65-95\% de conversão) em tempos variando de 7 a 25 min (Esquema 9).

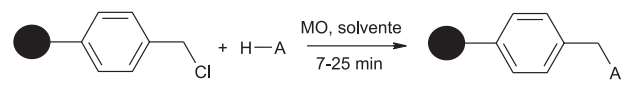

Esquema 9. Funcionalização da resina Merrifield

Bertena-Raboin et $a l .{ }^{7}$, na rota de síntese de derivados 5carboxamido- $N$-acetiltriptaminados já mencionada, realizaram a substituição eletrofílica do grupo trimetilsilil presente no anel indólico previamente sintetizado por iodo em $14 \mathrm{~min}$ em MO contra $24 \mathrm{~h}$ em refluxo (Esquema 3, reação "c").

O acoplamento de Suzuki ${ }^{14}$ na resina Rink Amide em MO foi realizado entre amidas aromáticas para-substituídas e diferentes ácidos organoborânicos no tempo de 3,8 min (Esquema 10). Outra $\mathrm{SN}_{\mathrm{Ar}}$, chamada de acoplamento de Songashira, foi realizada por Erdelyi e Gogoll ${ }^{15}$, entre aromáticos meta-substituídos, acoplados à resina Tentagel S RAM, e alquinos em um tempo médio de 20 min em um reator monomodo de MO (Esquema 11), com rendimentos variando de $84-99 \%$.

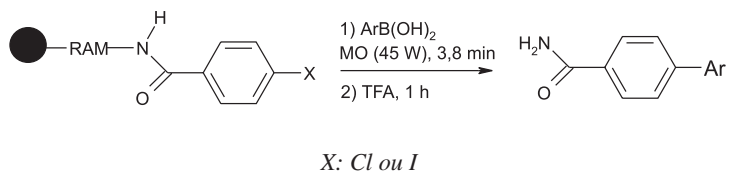

Esquema 10. Acoplamento de Suzuki

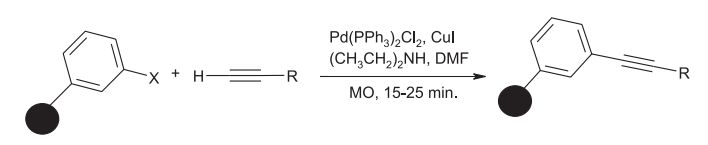

$X$ : Brou I

\section{Esquema 11. Acoplamento de Songashira}

A substituição nucleofílica de aminas secundárias em purinas iodo-substituídas, ligadas à resina Ameba-poliestireno, foi realizada recentemente ${ }^{16}$ (Esquema 12). O tempo relatado para esta reação foi de 30 min em MO, com rendimento de 45-59\%.

Kodadek et al., na rota de síntese de peptóides (Esquema 4) já mencionada anteriormente ${ }^{8}$, realizaram uma substituição no brometo

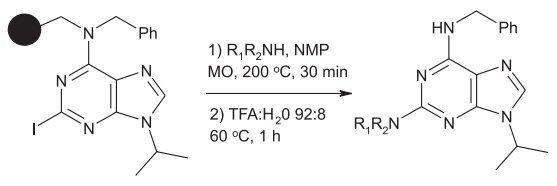

Esquema 12. Síntese de purinas

de alquila ligado à resina Rink Amide por uma amina primária em 2 x $15 \mathrm{seg}$. A rota inteira de síntese de um resíduo de peptóide tem tempo total de $1 \mathrm{~min}$ em $\mathrm{MO}$, contra $1,75 \mathrm{~h}$ em aquecimento convencional.

\section{Reações de ciclização}

Bertena-Raboin et $a .^{7}$ realizaram a formação elegante do anel indol com o auxílio de um alquino trimetilsilil substituído em presença de catalizador em 2 × 13 min em MO, contra 24 h de aquecimento convencional (Esquema 3, reação "b").

Dai et al. ${ }^{17}$, em outra rota de síntese de indóis, realizaram a etapa de ciclização intramolecular com auxílio de catalizador em 20 min utilizando MO (Esquema 13), com rendimentos variando entre 65$82 \%$.

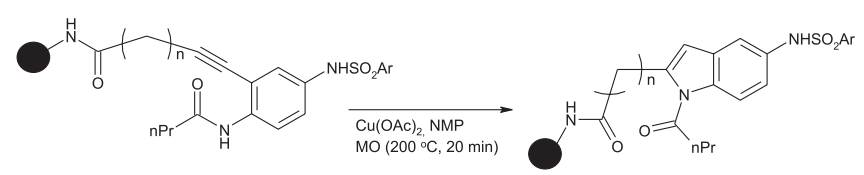

Esquema 13. Síntese de indóis

\section{Outras reações}

Reações Multi-Componente (RMC) são reações nas quais são adicionados três ou mais reagentes com grupos funcionais distintos em um meio reacional, obtendo de modo "one-pot" um aduto destes produtos. Uma vez que em apenas um passo se pode obter uma grande variabilidade química, esse tipo de reação atraiu grande atenção dos pesquisadores, principalmente na área da Química Combinatória. Nielsen e Hoel $^{18}$ realizaram a condensação de 4 componentes de Ugi em SOFS. Esta reação necessita da adição de um ácido, um aldeído, uma amina primária e uma isonitrila, o que foi realizado utilizando-se uma resina com um grupo amina como ligante (TentaGel S RAM) em 5 min em MO, com rendimentos de 24-96\%, gerando 18 análogos, quando os outros três componentes foram variados. Em aquecimento convencional o tempo relatado foi de $24 \mathrm{~h}$ (Esquema 14).
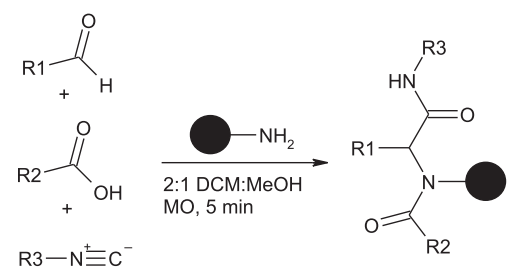

Esquema 14. Reação multi-componente de Ugi

Para se realizar esta reação, como já foi explicado, se necessita de quatro componentes, sendo a isonitrila talvez a mais difícil de se obter comercialmente. Bradley et al. ${ }^{19}$ desenvolveram uma síntese de isonitrilas a partir de uma amida $N$-substituída e uma resina com 
um grupamento clorossulfônico. O tempo de reação foi de 10 min em MO, contra $6 \mathrm{~h}$ de aquecimento convencional (Esquema 15).

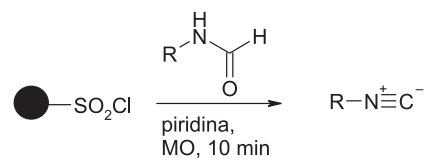

Esquema 15. Síntese de isonitrilas

\section{FUTURO PRÓXIMO}

O uso de reatores de microondas em laboratórios de síntese orgânica ainda não é rotineiro, em função do alto custo dos equipamentos, embora a sua vantagem como acelerador de reações químicas seja já bem conhecida e documentada.

Pode-se perceber claramente que esta tecnologia tem uma grande aplicação em processos onde o fator tempo é crítico. Por exemplo, na síntese de radiofármacos, onde o tempo de meia-vida do produto é um fator limitante para o seu uso, se a sua síntese fosse realizada em um tempo acelerado o seu tempo de uso seria prolongado ${ }^{4}$.

A síntese orgânica em fase sólida tem como característica principal a rapidez do processo como um todo, visto que a purificação dos intermediários e produtos é facilitada, contudo, o fator tempo nas reações também é essencial. Nesse caso, o uso de microondas visando a aceleração de sua produção torna-se uma alternativa viável e interessante para acelerar ainda mais o processo de descoberta de novas moléculas bioativas. Como foi demonstrado neste trabalho, já existem vários relatos, valendo-se tanto da síntese em fase sólida como em solução, para a produção de pequenas quimiotecas. Uma excelente estratégia sintética, adaptada às condições da SOFS, é o uso de reações multicomponente ${ }^{4}$, isto é, reações onde ocorre a reação de condensação ou conjugação de várias moléculas com grupos funcionais diferentes, como, por exemplo, a reação multicomponente de $\mathrm{Ugi}^{18}$. A variabilidade química que pode ser gerada a partir de reações como essa, onde cada molécula pode possuir grupamentos substituintes (R) diferentes (Esquema 14) é enorme. Estas reações em particular têm um tempo relatado em microondas de $5 \mathrm{~min}$, contra $24 \mathrm{~h}$ em aquecimento convencional.

Ressalta-se, também, que entre os pré-requisitos que a SOFS deve ter é importante que a síntese tenha um tempo viável e forneça bons rendimentos. Junte-se a isso a utilização das microondas, com todas as suas vantagens, a saber, tempos menores de reação, rendimentos e seletividade maiores, economia de energia e possibilidade de redução do uso de solventes, e as vantagens da SOFS, como a economia de tempo na purificação dos produtos intermediários de uma rota sintética, a facilidade de se aplicar a estratégia da Química Combinatória e a possibilidade de uma automatização integral do processo, tanto na SOFS como no reator de microondas, torna muito interessante para o químico orgânico a fusão destas técnicas.

\section{CONCLUSÕES}

$\mathrm{O}$ uso do reator de microondas em reações químicas apresenta evidências cada vez maiores de ser uma ótima ferramenta para a SOFS, devido aos relatos de tempos de reação menores e rendimentos maiores, entre outros benefícios.

Embora alguns fenômenos com esta técnica ainda não tenham sido totalmente explicados, aparentemente, a eficiência no aquecimento de meios reacionais (comparado com os métodos tradicionais) e a grande rapidez desse aquecimento são as principais causas da aceleração observada nas reações.
Especialmente na indústria farmacêutica, onde os fatores tempo e energia são cruciais no desenvolvimento e na produção de um novo fármaco, estas ferramentas combinadas apresentam um enorme potencial para revolucionar a pesquisa e a produção nesta área nos próximos anos.

A Síntese Orgânica em Fase Sólida torna-se, desta forma, uma metodologia ainda mais rápida e eficaz quando utilizada em conjunto com esta técnica, observando-se o decréscimo nos tempos de reação de horas para alguns minutos, quando não segundos. Observa-se também que o aquecimento muito rápido causado pelas microondas mantém a integridade das resinas, fator bastante positivo para a aplicação de SOFS.

\section{AGRADECIMENTOS}

Os autores agradecem aos Profs. E. R. de Oliveira (IQ/UFRGS) e P. Amaral (FF/UFRGS) pela leitura crítica do manuscrito e pelas sugestões. Agradecem também ao Prof. C. O. Kappe (Karl-FranzensUniversität, Graz/Áustria) pelo envio de algumas das referências.

\section{REFERÊNCIAS}

1. Merrifield, R. B.; J. Am. Chem. Soc. 1963, 85, 2149; Merrifield, R. B.; J. Org. Chem. 1964, 29, 3100; Frechet, J. M. J.; Tetrahedron 1980, 97, 489; Früchtel, J. S.; Jung, G.; Angew. Chem., Int. Ed. 1996, 35, 17; Thompson, L. A.; Elmann, J.; Chem. Rev. 1996, 96, 555; Hermkens, P. H.; Ottenheijm, H.; Rees, D.; Tetrahedron 1996, 52, 4527; Hermkens, P. H.; Ottenheijm, D.; Rees, D.; Tetrahedron 1997, 53, 5643; Marquardt, M.; Eifler-Lima, V. L.; Quim. Nova 2001, 24, 846.

2. Furlan, R. L. E.; Labadie, G. R.; Pellegrinet, S. C.; Ponzo, V. L; Quim. Nova 1996, 19, 411; Fenniri, H., ed.; Combinatorial Chemistry: A Practical Approach, Oxford University Press: Oxford, 2000; Dias, R. L. A.; Corrêa, A. G.; Quim. Nova 2001, 24, 236.

3. Gedye, R.; Smith, F.; Westaway, K.; Ali, H.; Baldisera, L.; Laberge, L.; Rousell, J.; Tetrahedron Lett. 1986, 27, 279. Giguere, R. J.; Bray, T. L.; Duncan, S. M.; Majetich, G.; Tetrahedron Lett. 1986, 27, 4945

4. Lindström, P.; Tierney, J.; Wathey, B.; Westman, J.; Tetrahedron 2001, 57, 9225; Sanseverino, A. M.; Quim. Nova 2002, 25, 660.; Barbosa, A. C. R. N.; Cruz, C. V. M. S.; Graziani, M. B.; Lorenzetti, M. C. F.; Sabadini, E.; Quim. Nova 2001, 24, 901; Pecoraro, E.; Davolos, M. R.; Jafelicci Jr., M.; Quim. Nova 1997, 20, 89; Wathey, B.; Tierney, J.; Lindström, P.; Westman, J.; Drug Disc. Today 2002, 7, 373; Kappe, C. O.; Am. Lab. 2001, 5, 13; Combs, A. P.; Dzierba, C. D. Em: Annual Reports in Medicinal Chemistry; Doherty, A. M., ed.; 37 ${ }^{\text {th }}$ ed., Academic Press: EUA, 2002; Hallberg, A.; Larhed, M.; Drug Disc. Today 2001, 6, 406; Loupy, A.; Perreux, L.; Tetrahedron 2001, 57, 9199; Caddick, S.; Tetrahedron 1995, 51, 10403; Kappe, C. O.; Curr. Opin. Chem. Biol. 2002, 6, 314; Chamberlain, R.; Lew, A.; Krutzik, P. O.; Hart, M.; J. Comb. Chem. 2002, 4, 95.

5. Kappe, C. O.; Stadler, A.; Eur. J. Org. Chem. 2001, 919.

6. Kappe, C. O.; Pérez, R.; Beryozkina, T.; Zbruyev, O. I.; Haas, W.; J. Comb. Chem. 2002, 4, 501 .

7. Berteina-Raboin, S.; Finaru, A.; Berthault, A.; Besson, T.; Guillaumet, G.; Org. Lett. 2002, 4, 2613.

8. Kodadek, T.; Olivos, H. J.; Alluri, P. G.; Reddy, M. M.; Salony, D.; Org. Lett. 2002, 4, 4057.

9. Stadler, A.; Yousefi, B. H.; Dallinger, D.; Walla, P.; Van der Eycken, E; Kaval, N.; Kappe, C. O.; Org. Process Dev. Res. 2003, 7, 707.

10. Amaral, P. A.; Dissertação de Mestrado, Universidade Federal do Rio Grande do Sul, Brasil, 2003

11. Hlasta, D. J.; Deng, Y.; Org. Lett. 2002, 4, 4017.

12. Scheeren, H. W.; Kuster, G. J.; Tetrahedron Lett. 2000, 41, 515.

13. Song, G.; Yang, H.; Peng, Y.; Qian, Y.; Tetrahedron Lett. 2001, 42, 9043.

14. Hallberg, A.; Larhed, M.; Lindeberg, G.; Tetrahedron Lett. 1996, 37, 8219.

15. Erdelyi, M.; Gogoll, A.; J. Org. Chem. 2004, 68, 6431.

16. Al-Obeidi, F.; Austin, R. E.; Okonya, J. F.; Bond, D. R. S.; Tetrahedron Lett. 2002, 43, 6169.

17. Dai, W.; Gun, D.; Sun, L.; Huang, X.; Org. Lett. 2003, 5, 2919

18. Nielsen, J.; Hoel, A. M. L.; Tetrahedron Lett. 1999, 40, 3941.

19. Bradley, M.; Launay, D.; Booth, S.; Clemens, I.; Merrit, A.; Tetrahedron Lett. 2001, 43, 7201. 